\title{
El clima de comunicación, la motivación y la satisfacción laboral en un proceso de atención primaria en Colombia
}

\author{
Edilson Bustamante \\ Universidad de Antioquia \\ (Medellín, Colombia)
}

\section{Palabras clave}

comunicación interna clima de comunicación motivación laboral satisfacción labora atención primaria

\begin{abstract}
Resumen
El proceso En Familia es una apuesta del Programa de Salud de la Universidad de Antioquia en Colombia que trabaja la atención primaria, desde la promoción y la prevención, y que cuenta con un equipo de empleados de más de 15 disciplinas. Esta investigación explora la relación entre la comunicación interna (clima de comunicación) y los componentes de motivación y satisfacción laboral de sus empleados, como indicadores de la apuesta que hace el proceso con sus públicos internos por potenciar desde dentro el trabajo con las familias beneficiarias. Se realizó un estudio descriptivo de corte transversal con una muestra integrada por 66 empleados de los 91 contratados durante el primer semestre de 2010. Se utilizó un cuestionario diseñado por el investigador. Los resultados permiten anotar que la relación de la comunicación con la motivación y satisfacción laboral es implícita, pues no tiende a discriminarse o sesgarse cada término, sino que se hace referencia a los tres de manera integrada. Se destacan además los siguientes elementos: el papel de los líderes; la comunicación cara a cara; la integración; el estímulo y la formación; la identificación; y las repercusiones de la comunicación interna, la motivación y la satisfacción laboral en el trabajo externo.
\end{abstract}

Esta investigación corresponde al trabajo final del autor en el Master Oficial en Gestión de la Comunicación en Instituciones Públicas y Estudios de Opinión de la Universidad Rey Juan Carlos (España). El autor agradece la colaboración y los aportes de Kelly Johana Santiago, Maylen Liseth Rojas, Maribel Cano, Diana Patricia Grajales, Susana Rodríguez, David Hernández García y del Programa de Salud de la Universidad de Antioquia.

Contacto para correspondencia: Edilson Bustamante. Email: edilsonbustamante@gmail.com. 


\section{Communication Climate, Motivation and Job Satisfaction in a Primary Care Process in Colombia}

\footnotetext{
Keywords

internal communication communication climate work motivation job satisfaction primary care
}

\begin{abstract}
The En Familia process has been carried out by the Health Program of the Universidad de Antioquia in Colombia, which focuses on the promotion of primary care and on prevention, and includes a team of employees specialized in over 15 different fields. This study explores the relation between internal communication (communication climate) and the components of employee motivation and job satisfaction, which are indicators of the effort made by the process regarding its internal audience so as to promote, from within, the work carried out with the beneficiary families. A descriptive cross sectional study was conducted with a sample composed of 66 out of the 91 employees hired during the first semester of 2010. A questionnaire designed by the researcher was used. The results show that the relation between communication, motivation and job satisfaction is implicit, since none of the terms are discriminated against or biased, but are referred to in an integrated manner. The following elements are also of note: the role carried out by leaders, face to face communication, integration, encouragement and training, identification, and the impact of internal communication, motivation and job satisfaction on external work.
\end{abstract}

\section{Cómo citar el artículo}

Bustamante, E. (2013). El clima de comunicación, la motivación y la satisfacción laboral en un proceso de atención primaria en Colombia. Revista de Comunicación y Salud, 3(1), pp. 35-49. DOI: http://doi.org/10.35669/revistadecomunicacionysalud.2013.3(1).35-49 


\section{Introducción}

En Colombia, la Ley 100 de 1993 reglamenta el Sistema General de Seguridad Social en Salud. Esta Ley contempla el funcionamiento del Sistema a través de Entidades Promotoras de Salud (EPS) como organismos encargados de administrar los recursos de salud para poblaciones determinadas. La Ley 647 de 2001 facultó a las universidades públicas para desarrollar sus propios Sistemas de Salud. Es el caso del Programa de Salud de la Universidad de Antioquia (PSUA), donde se desarrolló la presente investigación, y que opera como EPS para los empleados y jubilados de esta institución de educación superior pública. Desde el PSUA se ha buscado favorecer los procesos de promoción de la salud y prevención de la enfermedad, y ofrecer beneficios adicionales, apuntando a un modelo de salud que dista del actual modelo asistencial presente en la mayoría de EPS del país.

En busca de un modelo con estas características, el PSUA ha implementado desde hace varios años, dentro de su oferta de servicios, el proceso En Familia que trabaja desde la base de la atención primaria de salud, con acciones de promoción y prevención. Este proceso desarrolla acciones de asesoría y formación en diversas áreas (a través de visitas domiciliarias, talleres, medios de comunicación, entre otros) y ofrece la posibilidad de que los usuarios y sus familias participen en una red de grupos de ayuda mutua (redes de apoyo).

El proceso ha venido trabajando bajo un esquema donde se ha considerado a la comunicación como el eje transversal de la interacción entre los distintos actores. Prueba de ello es que En Familia es coordinado por la Facultad de Comunicaciones y participan además otras 15 unidades académicas ${ }^{1}$. Al tener en cuenta la comunicación en salud como eje transversal, el equipo de comunicaciones de En Familia desarrolla una estrategia completa dentro de la que se inscribe, entre otros asuntos, la comunicación interna, buscando mejorar el compromiso de cada integrante para que se refleje en su trabajo con las familias.

Se entiende por comunicación interna el proceso de gestión de los recursos humanos que actúa como elemento de motivación personal, fuente de transmisión y promoción de la cultura, y que fomenta la cohesión y optimiza el desarrollo, potenciando los comportamientos productivos (Somoza, 1993). La comunicación interna pretende, entre otras líneas objetivas, vincular el trabajo individual y los objetivos empresariales, transmitir la misión y los valores de una organización, mantener vivo el estímulo y la motivación en la tarea diaria, mejorar la satisfacción personal y mejorar la productividad (Berceruelo, 2011). Su objetivo final "es intangible, sea cual sea la naturaleza y el tamaño de la empresa: mejorar los resultados de la empresa y por lo tanto su rentabilidad" (Bannel, 1989: 49).

Desde esta perspectiva, esta investigación se centra en el clima de comunicación, entendido

1 En Familia nació en 2004 y es coordinado por la Facultad de Comunicaciones desde el 2006. Por tratarse de un proceso interdisciplinario, trabaja con profesores, egresados y estudiantes de otras quince unidades académicas de la Universidad de Antioquia. 
como la calidad del entorno comunicacional interno de la organización, experimentado subjetivamente por sus miembros (Dennis 1974), y que resulta de la interacción entre el clima psicológico -percepciones a nivel individual- y el clima organizacional -percepciones individuales homogéneas a nivel grupal- de los elementos e interacciones comunicativas (Hernández, 2008).

A partir del clima de comunicación se busca relacionar sus resultados con la motivación dentro del ámbito laboral, definida actualmente como el proceso que activa, orienta, dinamiza y mantiene el comportamiento de los individuos hacia los objetivos esperados (Toro, 2009), y con la satisfacción laboral como parte de una buena administración organizacional y que se da a partir de asuntos como el reto que implica el trabajo, el sistema de recompensas, las relaciones con los compañeros y las condiciones favorables para desarrollar el trabajo.

El proceso En Familia considera la comunicación interna (dentro de su visión de comunicación en salud) como uno de los pilares claves para alcanzar los objetivos planteados en el trabajo de promoción y prevención, acercándose a los postulados del marketing interno, entendido éste como un salto cualitativo en la dirección y gestión de los recursos humanos (Romeo, 2005), y siendo considerado "un modelo de intervención que permite a la empresa (organización) disponer permanentemente de un capital de recursos humanos capaz de dinamizar y de acompañar su propio desarrollo" (Quintanilla, 1988: 132).

Por eso, el objetivo de esta investigación es explorar la relación entre la comunicación interna (clima de comunicación) y los componentes de motivación y satisfacción laboral en los empleados del proceso En Familia, como indicadores de la apuesta que hace el proceso con sus públicos internos por potenciar desde dentro el trabajo de promoción y prevención que se realiza con las familias del PSUA. Las nuevas formas de concebir la salud, con rutas de trabajo como la atención primaria de salud (OMS, 1978) y con la filosofía de la promoción de la salud (OMS, 1986), requieren de nuevos actores que dinamicen los procesos de salud y nuevas formas de organizarse, y resulta evidente que la apuesta por los clientes internos (públicos internos) repercute en gran medida en el éxito de las acciones con los clientes externos (llámense usuarios, ciudadanos, personas, comunidades). De ahí la importancia de la comunicación interna en las organizaciones de salud, un área aún muy poco explorada en muchos de estos escenarios.

\section{Metodología}

Se realizó un estudio descriptivo de corte transversal. El universo de estudio estuvo conformado por los 91 empleados contratados por el Centro de Investigaciones y Extensión CIEC de la Facultad de Comunicaciones de la Universidad de Antioquia para laborar en el proceso En Familia durante el primer semestre de 2010 (enero a junio).

Sobre el universo de 91 trabajadores, y tomando como criterio un error máximo de $\pm 5 \%$, el muestreo probabilístico en población finita definió los 66 empleados que terminaron conformando la población de estudio. 
Se diseñó un cuestionario tomando como referencia el instrumento de García, Pacas y Ramírez (2006) para medir motivación y satisfacción laboral y la escala de Smidts et al. (2001), traducida al castellano por Hernández García (2008), para medir clima de comunicación interna. Sin embargo, se realizaron algunas modificaciones con el fin de obtener una información más completa y de adaptar el cuestionario a la población objeto de estudio. El cuestionario final, diseñado por el investigador, indagó:

a) las características sociodemográficas y laborales de los empleados;

b) motivación y satisfacción laboral; para medirlas se tomó como referencia el cuestionario de García, Pacas y Ramírez (2006);

c) comunicación; para investigarlo se contó con la escala "clima de comunicación" de Smidts et al. (2001);

d) y adicionalmente, se incluyeron dos interrogantes abiertos, los cuales indagaban sobre los aspectos que más motivan o desmotivan a nivel laboral en el proceso y sus razones, y las ideas que más se resaltan a nivel positivo o por mejorar de la comunicación interna.

El instrumento de García, Pacas y Ramírez (2006) para medir motivación y satisfacción laboral está conformado por 29 ítems a los que el encuestado debía indicar qué tan de acuerdo se encontraba con las afirmaciones allí expuestas, calificándolas como: de acuerdo, indeciso y en desacuerdo (5, 3 y 1 punto, respectivamente). La motivación es evaluada a través de 18 preguntas que dan pie a unos indicadores específicos: salario, prestaciones, seguridad, ambiente laboral y aspiraciones personales. Entretanto, la satisfacción es medida a partir de 11 preguntas relacionadas con importancia del trabajo, expectativas de exigencias del trabajo y expectativas entre esfuerzo y desempeño.

Por su parte, el instrumento de Smidts et al. (2001), traducido al castellano por Hernández García (2008), está conformado por 15 ítems medidos en una escala tipo Likert que va desde 1 (muy en desacuerdo) hasta 5 (muy de acuerdo). Dicho instrumento mide el clima de comunicación a partir de 3 dimensiones: apertura y confianza en la comunicación con la dirección, sentimiento de sentirse informado y apertura y confianza en la comunicación con los compañeros.

Para el procesamiento estadístico se realizó un análisis univariado y bivariado utilizando el paquete estadístico SPSS 18, y se aplicaron las pruebas estadísticas de asociación y correlación chi cuadrado y coeficiente de Spearman para explorar la relación entre las tres variables estudiadas.

\section{Resultados}

\subsection{Información sociodemográfica y laboral}

Al analizar la estructura de la población por edad se encontró que la mayoría de los participantes $(98,5 \%)$ eran adultos al momento de realizar la encuesta (personas entre los 20 y 44 años, según la Organización Mundial de la Salud). En cuanto al máximo grado de escolaridad alcanzado el porcentaje más alto correspondió a la categoría de carrera profesional, 40,9\%. El porcentaje de personas con posgrado estuvo al rededor del $11 \%$ (tabla 1 ). 
Tabla 1. Porcentajes variables sociodemográficas

\begin{tabular}{|c|c|c|c|}
\hline $\begin{array}{c}\text { Variables } \\
\text { sociodemográficas }\end{array}$ & Categorias & $n$ & $\%$ \\
\hline \multirow{3}{*}{ Ciclo vital } & Adolescente & 1 & 1,5 \\
\hline & Adultos jóvenes & 64 & 97,0 \\
\hline & Adultos medios & 1 & 1,5 \\
\hline \multirow{4}{*}{ Escolaridad } & Secundaria & 24 & 36,4 \\
\hline & $\begin{array}{l}\text { Técnica - } \\
\text { Tecnológica }\end{array}$ & 8 & 12,1 \\
\hline & $\begin{array}{l}\text { Carrera } \\
\text { profesional }\end{array}$ & 27 & 40,9 \\
\hline & Posgrado & 7 & 10,6 \\
\hline \multirow{2}{*}{ Dedicación laboral } & Medio tiempo & 39 & 59,1 \\
\hline & Tiempo completo & 27 & 40,9 \\
\hline \multirow{3}{*}{$\begin{array}{l}\text { Relación formación } \\
\text { académica con el } \\
\text { trabajo }\end{array}$} & Poca & 2 & 3,03 \\
\hline & Mucha & 21 & 31,8 \\
\hline & Totalmente & 43 & 65,2 \\
\hline
\end{tabular}

Fuente: elaboración propia

\subsection{Clima de comunicación}

Para los empleados del proceso En Familia, los cinco primeros ítems con mayores puntuaciones medias, correspondieron a las preguntas pertenecientes a la dimensión "apertura y confianza en la comunicación con la dirección". Por otro lado, las cinco puntuaciones medias más bajas en su mayoría correspondieron a la dimensión "sentimiento de sentirse informado".

\subsubsection{Puntuaciones medias}

Los promedios obtenidos en la muestra de los empleados del proceso En Familia en cada una de las dimensiones del clima de comunicación (de 1 a 5) están relacionados en la tabla 2. 
Tabla 2. Puntuaciones medias de las dimensiones del clima de comunicación

\begin{tabular}{|c|c|c|c|}
\hline \multirow{2}{*}{ Dimensiones del clima de comunicación } & \multicolumn{2}{|c|}{${ }^{\star} \mathrm{IC} 95 \%$} & \multirow{2}{*}{ Promedio } \\
\hline & LI & LS & \\
\hline Apertura y confianza en la comunicación con la dirección & 4,3 & 4,6 & 4,4 \\
\hline Sentimiento de sentirse informado & 3,8 & 4,1 & 4,0 \\
\hline $\begin{array}{l}\text { Apertura y confianza en la comunicación con los } \\
\text { compañeros }\end{array}$ & 4,1 & 4,4 & 4,2 \\
\hline
\end{tabular}

* IC: Intervalo de Confianza, LI: Límite Inferior, LS: Límite Superior.

Fuente: elaboración propia

\subsubsection{Grados del clima de comunicación}

Con el fin de medir los grados del clima de comunicación se empleó una clasificación tripartita de la variabilidad de los resultados en el instrumento. Se establecieron de esta manera tres valores posibles (alto, medio y bajo), según los posibles valores de respuesta. Con este propósito, se dividió el segmento de valores en tres zonas (superior, media e inferior), tomando como puntos de corte los percentiles 33 y 66 .

La implementación de esta clasificación en los empleados del proceso En Familia se expone en la tabla 3. Al respecto se destaca que dos de las tres dimensiones no presentaron puntuaciones en la categoría "bajo".

Tabla 3. Clasificación tripartita del clima de comunicación

\begin{tabular}{|l|l|c|c|}
\hline \multicolumn{1}{|c|}{$\begin{array}{c}\text { Dimensiones del clima de } \\
\text { comunicación }\end{array}$} & Valor & $\mathbf{n}$ & $\mathbf{\%}$ \\
\hline \multirow{2}{*}{$\begin{array}{c}\text { Apertura y confianza en la } \\
\text { comunicación con la dirección }\end{array}$} & Alto & 60 & 90,9 \\
\cline { 2 - 4 } & Medio & 6 & 9,1 \\
\cline { 2 - 4 } & Bajo & 0 & 0,0 \\
\hline \multirow{3}{*}{ Sentimiento de sentirse informado } & Alto & 43 & 65,2 \\
\cline { 2 - 4 } & Medio & 22 & 33,3 \\
\cline { 2 - 5 } & Bajo & 1 & 1,5 \\
\hline \multirow{2}{*}{$\begin{array}{c}\text { Apertura y confianza en la } \\
\text { comunicación con los compañeros }\end{array}$} & Alto & 53 & 80,3 \\
\cline { 2 - 4 } & Medio & 13 & 19,7 \\
\cline { 2 - 4 } & Bajo & 0 & 0,0 \\
\hline
\end{tabular}

Fuente: elaboración propia 


\subsubsection{Niveles de clima de comunicación}

En este caso se establecieron cinco diferentes niveles a partir de las posibles combinaciones en los percentiles 33 y 66 ya calculados y que fueron utilizados como puntos de corte. En este sentido los niveles de clima de comunicación se clasifican de la siguiente manera: muy bueno, bueno, ni bueno ni malo, malo, muy malo.

Al analizar la distribución de las frecuencias de los distintos niveles de clima de comunicación (tabla 4), se observa que ningún encuestado puntuó en dicho clima como "muy malo" o "malo".

Tabla 4. Distribución de las frecuencias de los niveles de clima de comunicación

\begin{tabular}{|l|l|l|}
\hline $\begin{array}{c}\text { Niveles de clima de } \\
\text { comunicación }\end{array}$ & $\mathbf{n}$ & $\mathbf{\%}$ \\
\hline Muy malo & 0 & 0,0 \\
\hline Malo & 0 & 0,0 \\
\hline Ni bueno ni malo & 1 & 1,5 \\
\hline Bueno & 8 & 12,1 \\
\hline Muy bueno & 57 & 86,4 \\
\hline Total & 66 & 100 \\
\hline
\end{tabular}

Fuente: elaboración propia

\subsubsection{Cálculo final}

Para el cálculo del clima de comunicación en los empleados del proceso En Familia se tuvo en cuenta la anterior clasificación, y se reagruparon algunos niveles para formar una nueva clasificación tripartita constituida por deseable (correspondiente a empleados que obtuvieron "muy bueno" y "bueno" en el nivel de clima de comunicación), aceptable (se tomaron los empleados que fueron clasificados en el nivel "ni bueno, ni malo"), indeseable (correspondiente a empleados que obtuvieron "malo" y "muy malo" en el nivel de clima de comunicación).

Se evidenció un clima de comunicación "deseable" en el 98,5\% (65) de los casos.

\subsection{Motivación y satisfacción laboral}

\subsubsection{Motivación laboral}

Se preguntó a las personas si consideraban que los beneficios económicos recibidos en el empleo satisfacían sus necesidades básicas, a lo que el $81,8 \%$ dijo estar de acuerdo, el 10,6\% estuvo en desacuerdo y un $7,6 \%$ de los encuestados estaban indecisos frente a dicha afirmación. 
Por otra parte, el 92,4\% de los encuestados estuvo en desacuerdo con la afirmación "creo que he elegido mal mi profesión". El porcentaje de personas indecisas en esta categoría fue del $3 \%$ y el porcentaje restante correspondió a las personas que consideraron haber hecho una mala elección de su carrera profesional

Otro de los ítems analizados fue el deseo de tener otro empleo en lugar del actual, con lo que el $81,8 \%$ de los encuestados estuvo en desacuerdo, el $16,7 \%$ se encontraba indeciso y solo un $1,5 \%$ estuvo de acuerdo con esta posición

La gran mayoría de los encuestados consideró que el reconocimiento social que se les tenía, en comparación al que se les tiene a otros profesionales, era adecuado a la función que desempeñaban, $84,8 \%$.

En el tema de la seguridad laboral, se observó que menos de la mitad de los encuestados dijo sentirse seguro y estable con su empleo actual, $45,5 \%$. Por otra parte, la totalidad de las personas participantes en este estudio, estuvo de acuerdo en que sus jefes mantenían buenas relaciones laborales con ellos.

Finalmente, teniendo en cuenta las especificaciones propias del instrumento, los resultados arrojaron que el $98,5 \%$ de los encuestados se encontraban "medianamente motivados", mientras que el $1,5 \%$ restante se ubicó en la categoría "muy motivado". Ningún participante se encontraba poco motivado al momento de la aplicación de la encuesta.

\subsubsection{Satisfacción laboral}

La gran mayoría de las personas seleccionadas $(98,5 \%)$ se sentían satisfechas porque consideraban que habían logrado contribuir en el área para la cual trabajaban. El $90 \%$ consideró que las retribuciones económicas que recibía en el trabajo estaban acordes con el esfuerzo que realizaban, mientras que un $13,6 \%$ de los encuestados afirmó sentirse subutilizado en el puesto que desempeñaba.

El $65,2 \%$ de los encuestados consideró que podría hacer mejor su trabajo, un 18,2\% se mostró indeciso, mientras que el $16,7 \%$ no identificó aspectos por mejorar. Por otra parte, se cuestionó acerca de la relación del trabajo con las expectativas personales de los trabajadores encontrándose una respuesta afirmativa en el $81,8 \%$ de los casos.

Finalmente, después de recategorizar los resultados del puntaje de satisfacción laboral en las 3 dimensiones sugeridas en el instrumento base (alta, mediana y baja satisfacción laboral), se observó que el $100 \%$ de los empleados del proceso se concentraron en la primera categoría "alta satisfacción laboral". 


\subsection{Preguntas abiertas}

Dentro de los aspectos que más motivan se encontró que un alto porcentaje se siente motivado por el proceso En Familia debido a la satisfacción personal y profesional que generan sus labores con las familias. Otro asunto tenido en cuenta por una gran mayoría, fueron las buenas relaciones interpersonales y construcciones interdisciplinarias que se generan en el interior del proceso. Otros asuntos destacados fueron el tipo de liderazgo, las buenas estrategias de comunicación interna, y el buen ambiente laboral.

Dentro de los aspectos que más desmotivan se encontró que lo que más afecta a todo el equipo de trabajo es la estabilidad laboral, por el tipo de contratación bajo la modalidad de prestación de servicios. Otro asunto manifestado por algunos de los encuestados fue el no cumplimiento de las metas debido a factores externos como el tiempo y la disposición de las familias para el trabajo.

En relación con los asuntos positivos de la comunicación interna se identificó que los encuestados, en su totalidad, señalaban la existencia de una buena comunicación interna, siendo los elementos más mencionados el cara a cara (el más preferido por los encuestados), las reuniones, los medios de comunicación internos, y los correos electrónicos. Destacan además el hecho de que el proceso cuente con espacios de encuentro, estímulo, integración $y$ formación entre sus integrantes.

En cuanto a los aspectos por mejorar de la comunicación interna, algunos encuestados manifestaron que en algunas ocasiones temas de interés han llegado en forma de rumor por parte de los compañeros.

\subsection{Exploración de la relación entre clima de comunicación, motivación y satisfacción laboral}

Con el fin de explorar la relación entre estas tres variables, se pretendía aplicar las pruebas estadísticas pertinentes (chi cuadrado de independencia y prueba de correlación de Spearman). Sin embargo, dado el comportamiento de estas variables, que más bien parecieran constantes, donde se encontró un $100 \%$ de empleados con "alta satisfacción laboral", un $98,5 \%$ "medianamente motivado" y otro 98,5\% calificando como "bueno" el clima de comunicación, dichas relaciones no pudieron explorarse.

\section{Discusión}

En general, los empleados del proceso En Familia expresan estar motivados y satisfechos por el tipo de trabajo que desempeñan y por el entorno laboral en el que se desenvuelven. Un factor que no favorece la motivación es el tipo de contratación, que no garantiza la estabilidad laboral ni ofrece los beneficios que tienen otros tipos de contratación. Sin embargo, el personal reconoce en la mayoría de los casos que la remuneración es suficiente y que su vinculación con el proceso va más allá de las ganancias económicas y se sitúa en otro tipo de ganancias 
a nivel profesional y personal, como la satisfacción con lo que hacen, el cumplimiento de sus expectativas profesionales y el reconocimiento social que les otorga. Esto ratifica que "el trabajador ya no busca solamente ganarse la vida, sino también expresar a través del trabajo su personalidad y experimentar satisfacción" (Gelinier, 1980: 261).

Uno de los asuntos que más incide tanto en la motivación como en la satisfacción laboral de los empleados es la visión estratégica de la comunicación, que se reconoce como esencial y oportuna dentro del proceso, generando un buen clima de comunicación y un buen clima laboral. La relación de la comunicación con la motivación y satisfacción laboral se encuentra implícita en cada una de las respuestas dadas por los encuentados, pues no tiende a discriminarse o sesgarse cada término, sino que en la mayoría de los casos se hace referencia a los tres de manera integrada. Esta unidad de conceptos da a entender que la comunicación es transversal a este proceso. De esta forma, se puede decir que la comunicación es un factor esencial que determina tanto la motivación como la satisfacción de los empleados, tal y como lo han demostrado otros estudios (Daly y Koprinek, 1982; Hernández, 2008).

Desde esta óptica, y teniendo en cuenta los resultados del trabajo de campo, se resaltan algunos aspectos propios de la comunicación interna con los empleados del proceso En Familia que influyen en su motivación y satisfacción laboral:

1. El papel de los líderes. Tanto en la medición del clima de comunicación como en las preguntas abiertas de motivación y satisfacción laboral, este fue un tema resaltado, destacando la visión que los empleados tienen de los líderes del proceso como profesionales cercanos, con los que se establecen buenas relaciones, y con un estilo de dirección participativo, lo que genera un ambiente de confianza e impulsa los sentimientos de motivación y pertenencia. Es bueno recordar que la manera en que los empleados perciben a la alta dirección influye en la satisfacción del trabajador (Goodman y Ruch, 1981) y que la comunicación vertical influye en gran medida en el compromiso de los integrantes de una organización (Postmes, Tanis y De Wit, 2001). Así, el éxito de una organización depende fundamentalmente de la profesionalidad, la dedicación, y la capacidad de comunicarse de los empleados y los líderes (Ferrer, 2000), y la política de comunicación interna se convierte en el indicador del estilo de liderazgo que se desarrolle en una determinada organización (Costa, 2011).

2. La comunicación cara a cara. Aunque los empleados reconocen que existen diversas formas de comunicación interna, incluidos los medios electrónicos y medios visuales como las carteleras, destacan la comunicación cara a cara como la forma de comunicación que más les atrae y que más beneficios otorga al proceso. Según los empleados, esta interacción comunicativa les da confianza, seguridad y claridad en las actuaciones, permite el debate y el consenso, y sienten que sus opiniones son tenidas en cuenta; además expresan que las interacciones cara a cara con sus compañeros y superiores inciden en gran medida en su motivación para el trabajo.

3. La integración. Los empleados rescatan los espacios que el proceso tiene para la formación, el encuentro, la integración y el trabajo en equipo, más aún si se tiene en cuenta que es un grupo conformado por una gran cantidad de disciplinas, miradas y formas de trabajar. Los distintos escenarios, pensados para la integración dentro del proceso, hacen que los 
empleados reconozcan en éstos grandes momentos para la construcción interdisciplinaria, para la generación de un buen clima laboral, para potenciar las buenas relaciones interpersonales que conducen a su vez a las buenas relaciones profesionales y a que se genere un ambiente de confianza para el trabajo colaborativo. Los comportamientos organizativos son el reflejo de una estructura social y, por tanto, dependiendo de cómo se opere con ella se pueden conseguir unos mejores o peores rendimientos laborales (Marqués, 2009). Una red con un alto grado de relaciones muy cercanas y con cierta carga emocional en el equipo repercutirá positivamente en el rendimiento del grupo (Balkundi y Harrinson, 2006). En resumen, la integración busca mejorar el clima de comunicación y fomentar la colaboración y el trabajo en equipo entre personas, áreas y niveles (Andrade, 2005).

4. El estímulo y la formación. Dentro de los escenarios de integración, los empleados encuentran espacios dedicados a la formación permanente del equipo, además reconocen la importancia de los procesos formativos que se gestan para el trabajo interdisciplinario y la formación en habilidades de comunicación necesarias para su labor con las familias. Asimismo destacan los procesos permanentes de estímulo y devolución que permiten por un lado mejorar los procesos, y por otro, motivarse más al sentirse importantes dentro de En Familia. En este sentido, se ha demostrado que las capacidades y comportamientos de los individuos pueden ser mejorados para convertirlos en el activo más importante de cualquier organización (Barney, 1991), lográndolo a través de la comunicación.

5. La identificación. Las respuestas muestran una alta identificación de los empleados con la cultura corporativa de En Familia, lo que conlleva a que muestren un alto orgullo y sentido de pertenencia por el proceso y un deseo de seguir perteneciendo a él. Esto se da debido a que han encontrado en el proceso una sintonía con su formación profesional, sus gustos, y sus expectativas profesionales y personales. Los empleados destacan que los procesos comunicativos han contribuido a aumentar su sentido de pertenencia. Asimismo subrayan los procesos de selección del personal, que conllevan que los perfiles seleccionados correspondan realmente con las necesidades del proceso (en cuanto a formación y filosofía de trabajo, en este caso de promoción de la salud y trabajo comunitario). La identificación permite orientar las acciones y decisiones de los empleados a partir de los principios que tienen establecidos respecto a la organización (Andrade, 2005).

6. Desde dentro hacia fuera. Sin lugar a dudas, la mayoría de los empleados reconoce que uno de sus mayores sentimientos de motivación y satisfacción se da en los resultados alcanzados en el trabajo real con las familias del proceso, y que indiscutiblemente los resultados se basan en gran parte en el trabajo interno, donde la comunicación juega un gran papel. Así, los empleados sienten que la motivación, la identificación con el proceso y su filosofía de trabajo, la formación, la información, y la integración, que se gestan al interior de En Familia, influyen decisivamente en los resultados finales del trabajo realizado con la población beneficiaria. Los empleados promueven a su organización en lo cotidiano, en el marco de sus contactos externos, personales y profesionales (Bartoli, 1992), donde transmiten sus motivaciones, convicciones y sus expectativas en cuanto a la realidad organizacional (Lingán, 2003). Por lo tanto, podría hablarse de que un empleado satisfecho es un cliente satisfecho y, desde esta óptica, los empleados y usuarios de los organismos de salud son actores que están en contacto permanente, y de ahí la importancia de que la comunicación e 
información al usuario la realicen profesionales motivados, satisfechos con su trabajo, cohesionados, formados y orgullosos de la institución para la cual trabajan (Costa, 2011).

Y si bien este estudio presenta limitaciones como el hecho de la no comprobación de la fiabilidad y concordancia inter e intraevaluador, al utilizar un instrumento construido por el investigador y al no estar validado, abre una puerta para posteriores estudios que permitan vislumbrar cómo desde la comunicación interna y su relación directa con la motivación y la satisfacción laboral, se pueden potenciar las acciones de promoción de la salud, una tarea que implica una labor desde dentro con empleados del sector sanitario como aliados estratégicos.

Variados estudios han demostrado la importancia de la comunicación interna en las organizaciones, destacando asuntos como su impacto en el compromiso organizacional (Welsch y LaVan, 1981; Guzley, 1992), en el desempeño de los equipos (Choi y Kim, 1999; Pincus, 1986), o su influencia en la productividad (Clampitt y Downs, 1993). En los escenarios de salud apenas se empieza a comprender el sentido y la importancia de ésta. En el caso de En Familia, que trabaja la promoción de la salud (puntualmente desde la perspectiva de la atención primaria y como una de las experiencias piloto en el país), y en todos los escenarios donde se trabaje por ésta (que deberían ser todos, incluyendo los hospitales y clínicas que actualmente son más referentes de la enfermedad que de la salud), la comunicación interna tiene que trazar una ruta muy importante para lograr informar, formar, integrar, crear una imagen, e identificar a todos sus empleados con la filosofía de trabajo que implica promocionar la salud (Bustamante, 2012). Sólo así es posible, desde los escenarios de salud, pensar en crear ambientes favorables, desarrollar habilidades y capacidades en las personas, reforzar la acción comunitaria, reorientar los servicios de salud y trabajar por el establecimiento de políticas públicas saludables, pilares básicos de la promoción de la salud.

\section{$\overline{\text { Referencias }}$}

Andrade, Horacio. (2005). Comunicación organizacional interna: proceso, disciplina y técnica. España: Netbiblo.

Balkundi, Prasad y Harrinson, David. (2006). Ties, Leaders and Time in Teams: Strong Inference about Network Structure's Effects on Team Viability and Performance. Academy of Management Journal. Vol. 49, n 1, pp. 49-68. doi: 10.5465/AMJ.2006.20785500.

Bannel, Yves. (1989). Comunicación y marketing interno: la revolución empresarial de hoy. Madrid: Gramar.

Barney, Jay B. (1991). Firm Resources and Sustained Competitive Advantages. Journal of Management. Vol. 17, $\mathrm{n}^{\circ} 1$, pp. 99-120. doi: 10.1177/014920639101700108.

Bartoli, Annie. (1992). Comunicación organizacional: la organización comunicante y la comunicación organizada. Barcelona: Ediciones Paidós.

Berceruelo, Benito. (2011). Comunicación interna en la empresa: claves y desafíos. España: Wolters Kluwer. 
Bustamante, Edilson. (2012). La promoción de la salud desde la comunicación interna. Revista de Comunicación y Salud. Vol. 2, n² 2, pp. 79-90.

Choi, Jin Nam y Kim, Myung Un. (1999). The Organizational Application of Groupthink and its Limitations in Organization. Journal of Applied Psychology. Vol. 84, ${ }^{\circ} 2$, pp. 297-306. doi: 10.1037/0021-9010.84.2.297.

Clampitt, Phillip G. y Downs, Cal W. (1987). Communication Satisfaction: A Review of the Literature. Monografía inédita. University of Kansas. Kansas.

Costa, Carmen. (2011). La comunicación en el hospital: la gestión de la comunicación en el ámbito sanitario. Sevilla: Comunicación Social, ediciones y publicaciones.

Daly, John A. y Korinek, J. (1982). Organizacional Communications: A Review Via Operationalizations. En Greenbaum, Howard H. y Falcione, Raymond L. (Eds.). Organizational Communication: Abstracts, Analysis, and Overview. Beverly Hills: Sage Publications.

Dennis, Harry S. (1974). A Theoretical and Empirical Study of Managerial Communications Climate in Complex Organizations. Tesis doctoral inédita. Purdue University. Indiana.

Ferrer, Joan (2000). La comunicación interna y externa en la empresa. Barcelona: Edición Privada.

García, Claudia; Pacas, Kenny y Ramírez, Linda Ruth. (2006). Estudio expost facto sobre la motivación y la satisfacción laboral. Monografía inédita. Universidad de El Salvador. EI Salvador.

Gelinier, Octave. (1980). La nueva dirección de la empresa. Madrid: APDO.

Goodman, R. y Ruch, R. (1981). In the Image of the CEO. Public Relations Journal. Vol. 37, $\mathrm{n}^{\circ}$ 2, pp. 14-19.

Guzley, Ruth M. (1992). Organizational Climate and Communication Climate: Predictors of Commitment to the Organization. Management Communication Quarterly. Vol. 5, $\mathrm{n}^{\circ}$ 4, pp. 379402. doi: $10.1177 / 0893318992005004001$.

Hernández, David. (2008). La relación entre el sistema formal de comunicación interna, el clima de comunicación y la identificación de los empleados en las organizaciones. Tesis doctoral inédita. Universidad de Barcelona. España.

Ley 647/2001, de 28 de febrero, del Carácter Especial del Régimen de las Universidades Estatales u Oficiales. Congreso de la República de Colombia.

Ley 100/1993, de 23 de diciembre, del Sistema General de Seguridad Social en Salud. Congreso de la República de Colombia.

Lingán, Jéssica (2003). Implicaciones conceptuales y metodológicas de la comunicación estratégica en la gestión de la imagen corporativa interna de los servicios sanitarios. El caso del hospital Valle del Hebrón. Tesis doctoral inédita. Universidad Autónoma de Barcelona. España. 
Marqués, María del Pilar. (2009). Influencia de las redes sociales en el rendimiento de las organizaciones: un estudio del sector sanitario. Tesis doctoral inédita. Universidad de León. España.

Organización Mundial de la Salud. (1986). Carta de Otawa para la promoción de la salud. Disponible en http://www.paho.org/spanish/hpp/ottawachartersp.pdf. Recuperado el 1 de agosto de 2012.

Organización Mundial de la Salud. (1978). Declaración de Alma-Ata sobre Atención Primaria de Salud APS. Disponible en http://whqlibdoc.who.int/publications/9243541358.pdf. Recuperado el 1 de agosto de 2012.

Pincus, J. David. (1986). Communication Satisfaction, Job Satisfaction, and Job Performance. Human Communication Research. Vol. 12, nº 3, pp. 395-419. doi: 10.1111/j.1468-2958.1986.tb00084.x.

Postmes, Tom; Tanis, Martin y De Wit, Boudewijn. (2001). Communication and Commitment in Organization: A Social Identity Approach. Group Processes and Intergroup Relations. Vol. 4, $\mathrm{n}^{\circ} 3$, pp. 227-246. doi: 10.1177/1368430201004003004.

Quintanilla, Ismael. (1988). Psicología y marketing. Evaluación de la conducta del consumidor y otras extensiones. Valencia: Promolibro.

Romeo, Marina. (2005). Hacia la comunicación global, marketing interno. En Almerana, Jaume (Coord.). Comunicación interna en la empresa. Barcelona: Editorial UOC.

Smidts, Ale; Pruyn, Ad T.H. y Riel, Cees. (2001). The Impact of Employee Communications and Perceived External Prestige on Organization Identification. Academy of Management Journal. Vol. 49, nº 5, pp. 1.051-1.062. doi: 10.2307/3069448.

Somoza, Fernando. (1993). Director de comunicación. España: Ediciones Edipo.

Toro, Fernando. (2009). Clima organizacional. Una aproximación a su dinámica en la empresa latinoamericana. Medellín: Centro de Investigación en Comportamiento Organizacional (CINCEL).

Welsch, Harold P. y LaVan, Helen. (1981). Interrelationships between Organizational Commitment and Job Characteristics, Job Satisfaction, Professional Behavior and Organizational Climate. Human Relations. Vol. 34, $\mathrm{n}^{\circ}$ 12, pp. 1.079-1.089. doi: 10.1177/001872678103401205. 